\title{
Single-Photon Interference due to Motion in an Atomic Collective Excitation
}

\author{
D. J. Whiting, ${ }^{*}$ N. Šibalić, J. Keaveney, C. S. Adams, and I. G. Hughes \\ Joint Quantum Centre (JQC) Durham-Newcastle, Durham University, \\ Department of Physics, South Road, Durham, DHI 3LE, United Kingdom
}

(Received 3 February 2017; published 22 June 2017)

\begin{abstract}
We experimentally demonstrate the heralded generation of bichromatic single photons from an atomic collective spin excitation (CSE). The photon arrival times display collective quantum beats, a novel interference effect resulting from the relative motion of atoms in the CSE. A combination of velocityselective excitation with strong laser dressing and the addition of a magnetic field allows for exquisite control of this collective beat phenomenon. The present experiment uses a diamond scheme with near-IR photons that can be extended to include telecommunications wavelengths or modified to allow storage and retrieval in an inverted- $Y$ scheme.
\end{abstract}

DOI: 10.1103/PhysRevLett.118.253601

I. Introduction.-Quantum-state engineering is of critical importance to the development of quantum technologies. Atomic media are an attractive option for realizing these technologies [1], providing well-defined optical transitions, long coherence times, frequency-matched high-brightness single-photon sources [2,3], quantum memories [4-6] and repeaters [7], coherent control protocols based on slow light and adiabatic following [8], and strong nonlinearities that produce controllable phase shifts [9]. While there are clear advantages over solid-state approaches [10], the technological complexity of typical cold-atom experiments presents a challenge for scaling and wider application.

In contrast to cold-atom systems, thermal atomic vapor experiments provide a reproducible and scalable hardware platform. Their use has enabled the development of many practical devices including chip-scale atomic clocks [11], brain sensors [12], and microwave electrometers [13]. However, the inability to address individual atomic states in a controlled manner, due to multilevel degeneracy and motional broadening, inhibits their wider use in quantumstate engineering applications. Optical pumping is conventionally used for initial state preparation, and buffer gases $[14]$ and antirelaxation coatings $[15,16]$ can be employed to mitigate decoherence processes for ground-state atoms. However, for schemes involving excited states [17] or thin cells [18], these methods usually cannot be applied. An alternative solution is to apply a strong magnetic field that resolves the multilevel degeneracy. This method has recently been shown to simplify nonlinear atom-light interactions in thermal vapors, resulting in the enhanced control of electromagnetically induced transparency [19]

Published by the American Physical Society under the terms of the Creative Commons Attribution 4.0 International license. Further distribution of this work must maintain attribution to the author(s) and the published article's title, journal citation, and DOI. and absorption [20]. Another major challenge facing the application of thermal atomic vapors to quantum-state engineering is motion-induced dephasing [21] because of the broad atomic velocity distribution. It is therefore interesting to consider novel quantum states that exploit this motion, for example, when a single excitation is stored in an entangled state of two atoms with relative motion. This state was discussed theoretically in the 1970s but was deemed "impossible to observe directly" [22] in thermal vapors due to the wide spread of velocities rapidly washing out the spatial correlations between atoms.

In this Letter, we demonstrate a method to engineer this type of collective state in a thermal atomic vapor. The prepared state consists of a single excitation as a robust collective superposition of two velocity classes, whose coherent nature is demonstrated by measuring collective quantum beats [22]. The single excitation is emitted as a single photon with two frequencies. At present, there is much interest in these "bichromatic" photons, as they could be used to entangle spatially separated quantum memories or perform spectroscopy with small numbers of photons $[23,24]$. Combining the application of a large magnetic field and strong laser dressing in a velocity-selective laddertype excitation, we demonstrate excellent control over the state preparation.

II. State preparation.-During the state preparation, a strong magnetic field allows individual control over the internal atomic states, and a ladder-type excitation with strong laser dressing allows the tunable selection of the external (motional) states. A magnetic field $(B=0.6 \mathrm{~T})$ splits the atomic states according to their projection of spinorbit coupling $m_{J}$, by energy $m_{J} \mu_{B} B$, where $\mu_{B}$ is the Bohr magneton. This field, provided by permanent neodymium magnets, separates the optical transitions of the atom by more than their Doppler-broadened linewidth $[25,26]$. A pump laser can then be tuned to address only those atoms from the ensemble that are in the chosen $m_{J}$ state, $|g\rangle$, 
reducing the internal degrees of freedom of the system to four coupled levels [Fig. 1(a), inset]. A ladder-type excitation scheme with copropagating pump and coupling lasers [Fig. 1(a)] selects a narrow group of resonant atoms from the broad velocity distribution. A strong coupling laser dresses the bare atomic states $|a\rangle$ and $|b\rangle$, allowing the simultaneous excitation of two narrow velocity groups (with well-defined phases) satisfying the condition $2 k v_{z}=$ $\frac{1}{2}\left(\Delta_{c} \pm \sqrt{\Delta_{c}^{2}+2 \Omega_{c}^{2}}\right)$ (Fig. 1). These two groups correspond to the dressed states $\left|d_{1}\right\rangle$ and $\left|d_{2}\right\rangle$ in Fig. 1(a), inset. By choosing the detuning $\Delta_{c}$ and driving strength $\Omega_{c}$ of the coupling laser, with wave vector $k$, one can choose the velocities $v_{z}$ of the two excited velocity classes. For a negatively (red-) detuned coupling laser, these correspond to one nearly stationary group and one moving away from the detector [Fig. 1(b)].

A single collective excitation is produced by heralding on the spontaneous decay of the excited atoms. The herald photon maps the instantaneous relative phase of the atoms [Fig. 1(b)], from the steady state under strong laser driving, into the excited state $|e\rangle$. Since the strong driving preferentially selects two atomic velocity classes, the photon detection heralds the coherent splitting of the single excitation into these two velocity groups. The driving lasers and the herald and signal output channels fulfill the wave-matching condition as in usual diamond fourwave mixing schemes $[27,28]$. Because of this, the single excitation takes the form of a spin wave, picking out a preferential output direction for the collective emission of the signal photon [29]. Because of the atomic motion, the emission from the moving group of atoms will be Doppler shifted with respect to that of the stationary atoms [car and house in Fig. 1(c)]. This frequency shift leads to interference and the observation of beats in the signal emission [Fig. 1(d)], demonstrating the persistence of coherence in the single excitation split between two velocity groups. In contrast to usual quantum beats, that originate due to state superposition within the single-atom structure [30-32], these beats originate due to a superposition of atoms with different velocities being in the same internal excited state $|e\rangle$ [Fig. 1(e)]. Beating of light fields emitted by two groups of atoms with different velocities has previously been observed in superradiant emission from thermal ensembles after pulsed excitation [33]. However, these superradiant beats cannot be observed on a single-photon level, since which-path information is stored in the excited state regarding which atoms decay in the process; one could, in principle, check, for each emitted photon, which velocity class is in the excited state. Finally, we note that singlephoton beats can be observed in cold atoms (only one velocity class) by using an additional laser to dress the levels involved [34,35].

III. Experimental details.-Experimentally we use ${ }^{87} \mathrm{Rb}$ atoms in a diamond scheme with energy levels (a)
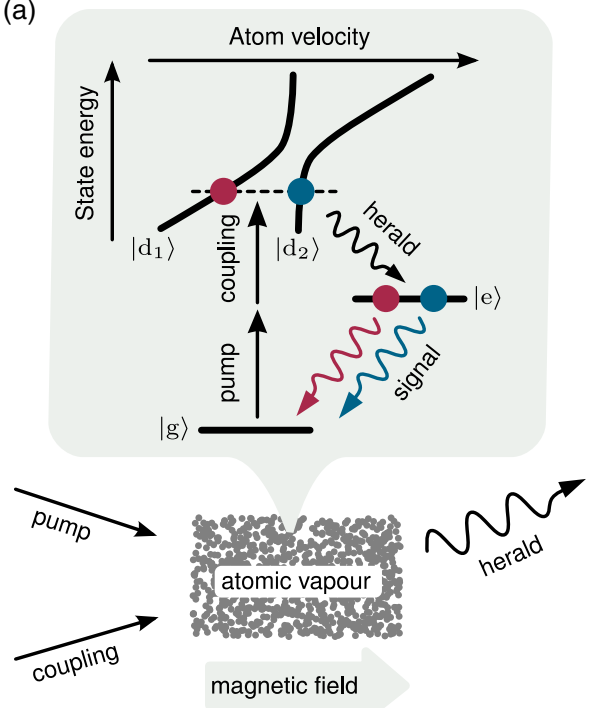

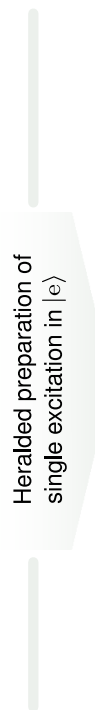

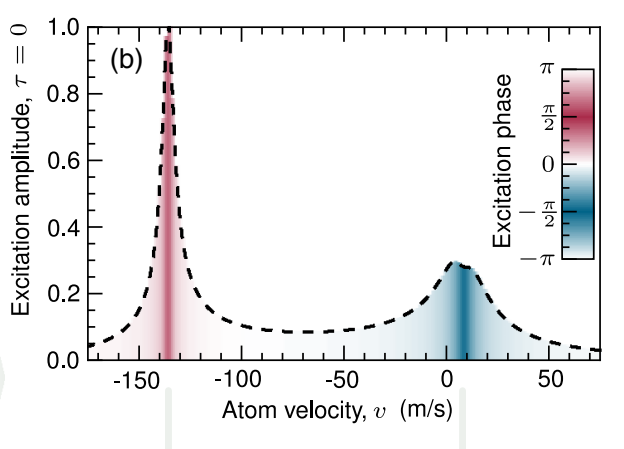

(c)

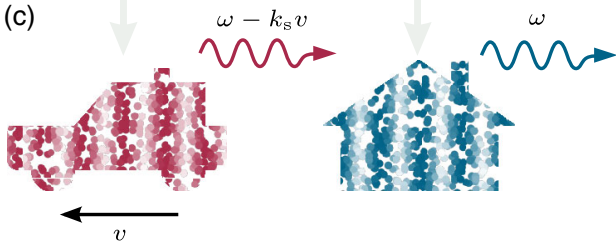

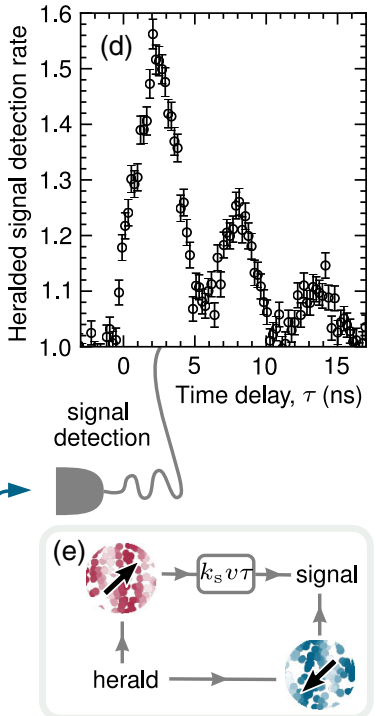

FIG. 1. Preparation of a single excitation as a collective superposition of two atomic velocity groups. (a) A thermal vapor of ${ }^{87} \mathrm{Rb}$ is continuously driven by weak pump- and strong coupling-laser beams. A strong magnetic field of 0.6 T, applied with permanent magnets, simplifies the internal level structure by isolating only the four levels shown in the inset (in the semidressed picture). The coupling laser dresses the atoms, so that atoms with two different velocities (shown in red and blue in the inset) are preferentially excited. The detection of a "herald" photon heralds the preparation of a single excitation in level $|e\rangle$ in the form of a spin wave. (b) The excitation is mostly split among two velocity classes, one stationary and one moving away from the signal detector in (c), with an initial phase difference of $\pi$. Because of the Doppler effect, light emitted from these two classes of atoms will be shifted in frequency, causing beats in the signal photon detection (d). The beats demonstrate that this setup forms an interferometer (e), where the detection of a herald photon coherently splits a single excitation and stores it in atoms moving at two different velocities, before recovering the excitation in a common signal channel. 


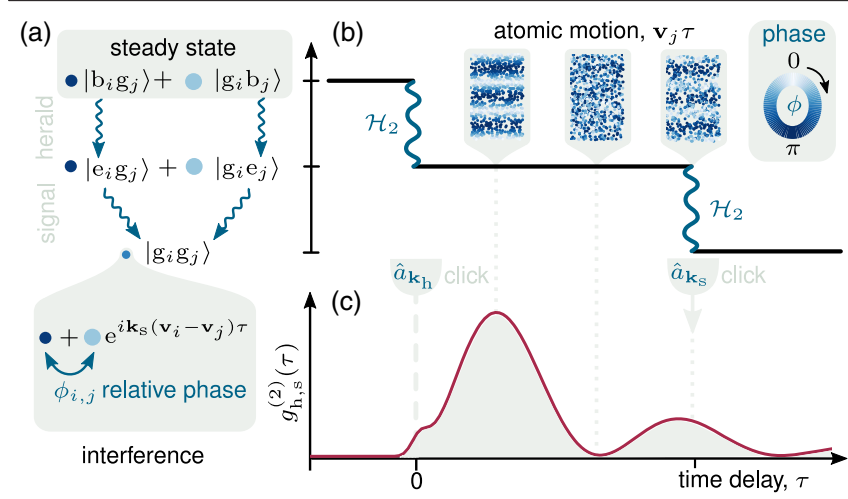

FIG. 2. Collective decay leading to beats. (a) Continuous driving prepares the system in a steady state, where atoms $i$ and $j$ (the sum over all possible $i, j$ is implied) are in a superposition of ground $|g\rangle$ and bare $|b\rangle$ states. Herald detection maps the steady-state amplitudes and phases (indicated by the size and color of the circles) into a superposition of excited states $|e\rangle$. (b) Subsequently, the relative phase of the atoms evolves due to motion (see the insets for the spin-wave evolution in space with color coding of the relative phase; the $B$-field orientation is vertical). Since both atoms end up in the same ground state after emitting the signal photon, the emission amplitudes add coherently and a time-dependent factor appears in the collective signal [bottom of (a)]. (c) This interference leads to beats in the probability of directional signal photon emission over time, $\tau$.

denoted $|g\rangle=5 S_{1 / 2}\left(m_{J}=1 / 2\right),|a\rangle=5 P_{3 / 2}\left(m_{J}=3 / 2\right)$, $|b\rangle=5 D_{3 / 2}\left(m_{J}=1 / 2\right)$, and $|e\rangle=5 P_{1 / 2}\left(m_{J}=-1 / 2\right)$. Continuous-wave pump and coupling fields are tuned to the $|g\rangle \rightarrow|a\rangle$ and $|a\rangle \rightarrow|b\rangle$ resonances at 780 and $776 \mathrm{~nm}$, respectively. The pump and coupling fields, with an angular separation of $10 \mathrm{mrad}$, are focused to $50 \mu \mathrm{m}\left(1 / e^{2}\right.$ waists $)$ and overlapped at the center of a $2 \mathrm{~mm}$ long atomic vapor cell. The cell, containing rubidium (isotopic abundance $98 \%{ }^{87} \mathrm{Rb}$ and $2 \%{ }^{85} \mathrm{Rb}$ ), is heated to $90{ }^{\circ} \mathrm{C}$. The cell also contains buffer gasses which contribute an additional broadening of $7 \mathrm{MHz}$ to the $5 S \rightarrow 5 P$ transitions and $13 \mathrm{MHz}$ to the $5 P \rightarrow 5 D$ transitions. The pump and coupling powers are $4 \mu \mathrm{W}$ and $40 \mathrm{~mW}$, respectively, which correspond approximately to Rabi frequencies of $\Omega_{p} / 2 \pi=$ $34 \mathrm{MHz}$ and $\Omega_{c} / 2 \pi=270 \mathrm{MHz}$. The herald and signal photons are spontaneously emitted on the transitions $5 D_{3 / 2}\left(m_{J}=1 / 2\right) \rightarrow 5 P_{1 / 2}\left(m_{J}=-1 / 2\right) \rightarrow 5 S_{1 / 2}\left(m_{J}=\right.$ $1 / 2)$ at 762 and $795 \mathrm{~nm}$, respectively. In this configuration, the generated photons are emitted in the forward direction to fulfill the phase-matching criterion $\mathbf{k}_{p}+\mathbf{k}_{c}=\mathbf{k}_{h}+\mathbf{k}_{s}$. After being separated from the pump light by narrow-band interference filters and polarization filtering (see Supplemental Material [36]), the generated photons are collected into single-mode optical fibers and detected by avalanche photodiodes. A timing card with a 27 ps resolution records the photon detection times which are used to calculate the histogram of herald-signal coincidence events, $G_{h, s}^{(2)}(\tau)$, as a function of time delay $\tau$ between herald and signal detections. The normalized herald-signal correlation function is calculated as $g_{h, s}^{(2)}=G_{h, s}^{(2)}(\tau) /$ $\left(r_{h} r_{s} \Delta \tau T\right)$, where $r_{h, s}$ are the count rates on the herald and signal detectors, $\Delta \tau$ is the histogram bin width, and $T$ is the total time over which counts were recorded. Figure 1(d) shows the resulting herald-signal correlation function under these conditions with a coupling laser detuned by $\Delta_{c} / 2 \pi=330 \mathrm{MHz}$.

IV. Theoretical model.-The probability of detecting a signal photon a time $\tau$ after heralding depends on the initial relative phase of the two velocity groups and the speed difference in the signal detector direction. To understand the process that sets the initial relative phase and the subsequent phase evolution of the atomic medium, consider an ensemble of atoms enumerated by $j$ in the basis $\otimes_{j}\left|\alpha_{j}, \mathbf{r}_{j}, \mathbf{v}_{j}\right\rangle \otimes\left|\hat{n}_{\mathbf{k}_{h}}\right\rangle \otimes\left|\hat{n}_{\mathbf{k}_{s}}\right\rangle$, where $\alpha \in\{g, a, b, e\}$ denotes the atomic state and $\hat{n}_{\mathbf{k}_{h}, \mathbf{k}_{s}}$ the occupation of the two decay modes corresponding to the herald and signal wave vectors $\mathbf{k}_{h, s}$. The atomic dynamics, dominated by evolution under strong laser driving and spontaneous decay to all other free modes, brings the system to the stationary state described by the density matrix $\sum_{i} c_{i}|\psi\rangle\langle\psi|$ [Fig. 2(a)]. Cascaded spontaneous four-wave mixing emission, due to the weak coupling $\mathcal{H}_{2}$ to the herald and signal modes, can be treated as a perturbative correction to the dynamics. The detection of a herald photon, $\hat{a}_{\mathbf{k}_{h}}$, therefore projects the system state into the collective spin wave

$$
\hat{a}_{\mathbf{k}_{h}} \mathcal{H}_{2}|\psi\rangle \propto \sum_{j} a_{j} e^{-i\left(k_{h}-k_{c}-k_{p}\right) z_{j}}\left|\ldots e_{j} \ldots\right\rangle,
$$

where $k_{h}, k_{c}$, and $k_{p}$ are the herald, coupling, and pump mode wave vectors, respectively, and factors $a_{j}$ depend on the atomic velocity component $v_{z}$. Since the herald detection is broadband, the projection is into a state where a single excitation $\left|e_{j}\right\rangle$ is in a superposition of different velocity classes.

During the subsequent time $\tau$, before the emission of the signal photon, the phase of the state given by Eq. (1) will not change, since state $\left|e_{j}\right\rangle$ is decoupled from the strong laser driving. However, the amplitude of this state will be reduced by $\exp (-\gamma \tau)$ due to spontaneous emission to other spatial modes and homogeneous dephasing mechanisms (e.g., collisions with buffer gasses). Upon the decay of $\left|e_{j}\right\rangle$ under $\mathcal{H}_{2}$ [Fig. 2(b)], the detection of the signal photon $\hat{a}_{\mathbf{k}_{s}}$ is also broadband and therefore does not differentiate between emission from different velocity classes. Therefore, no which-path information is measured. Emission from different velocity classes will, due to atomic motion [Fig. 2(b), insets], have a frequency shift of $k_{s} v_{z}$. This can give rise to beats in the signal photon detection [Fig. 2(c)], provided that no information is left in the medium about which atom emitted the photon. All states where the two atoms, labeled $i$ and $j$, are in the superposition of ground and excited state $c_{1}(t)\left|g_{i} e_{j}\right\rangle+$ $c_{2}(t)\left|e_{i} g_{j}\right\rangle$ fulfill that condition, since after cascaded herald 
and signal emission (time $\tau$ later) they end up in the same state $\left|g_{i} g_{j}\right\rangle$, where the amplitude shows interference between the two possible paths $c_{1}(t+\tau)+c_{2}(t+\tau)$ [bottom of Fig. 2(a)]. From this consideration, we see that the initial phase of the signal emission from the velocity class $v_{z}$ will be set by the stationary value of the singleatom coherence element $\rho_{b q}\left(v_{z}\right)$ between the states $|b\rangle$ and $|g\rangle$ for the corresponding velocity. Integrating over all the velocity classes, weighted according to their probabilities given by the Maxwell-Boltzmann distribution $f\left(v_{z}\right)$, one obtains (see Supplemental Material [36]) the two-photon correlation function $\left\langle\hat{a}_{\mathbf{k}_{s}}^{\dagger} \hat{a}_{\mathbf{k}_{s}} \hat{a}_{\mathbf{k}_{h}}^{\dagger} \hat{a}_{\mathbf{k}_{h}}\right\rangle_{\tau}=|\Psi|^{2}$, where

$$
\Psi \propto \int_{v_{z}} d v_{z} f\left(v_{z}\right) \rho_{b g}\left(v_{z}\right) \exp \left[-\left(\gamma+i k_{s} v_{z}\right) \tau\right] .
$$

We note that this calculation includes only the contribution from correlated decays and ignores the background of uncorrelated photon counts produced by other events (see Supplemental Material [36]). This gives the normalized joint-detection probability for the herald and signal photons, as defined by Glauber's theory [37], $g_{h, s}^{(2)}(\tau)=1+c|\Psi|^{2}$, where the constant of proportionality $c$ accounts for the uncorrelated background and is included as a free parameter in the model.

$V$. Experimental results. - The developed theoretical model agrees very well with the temporal correlation data over a wide range of parameters (Fig. 3). The model is fit to the data using chi-squared minimization [38] with common fit parameters for all data sets displayed. This agreement demonstrates the excellent understanding and control of the state preparation achieved in our experiment and compares very favorably to the cases without control over the initial state, like recent experiments in pulse-seeded four-wave mixing $[39,40]$. The observed lifetime of the collective coherence is on the order of the excited-state lifetime. During this coherence time, atoms in different velocity groups can be independently perturbed by external fields, e.g., by exploiting their Doppler-shifted optical resonances with coherent driving. An applied perturbation would imprint a different phase on the excitation stored in each velocity group, which could be directly measured by the accompanying change in the herald-signal correlation. In the future, an inverted- $Y$ scheme could be used, combining a typical $\Lambda$ scheme with an additional laser that strongly dresses the intermediate state [41]. This would enable the storage and deterministic retrieval of the split single photon, due to the long-lived ground-state coherences and longer spin-wave period [42]. During the storage time, the usual qubit rotation operations could be performed by applying off-resonant driving that imprints a relative phase via the ac-Stark shift.

The heralded single photon has primarily two frequency components, the frequencies and amplitudes of which are tunable via the coupling laser parameters and the magnetic

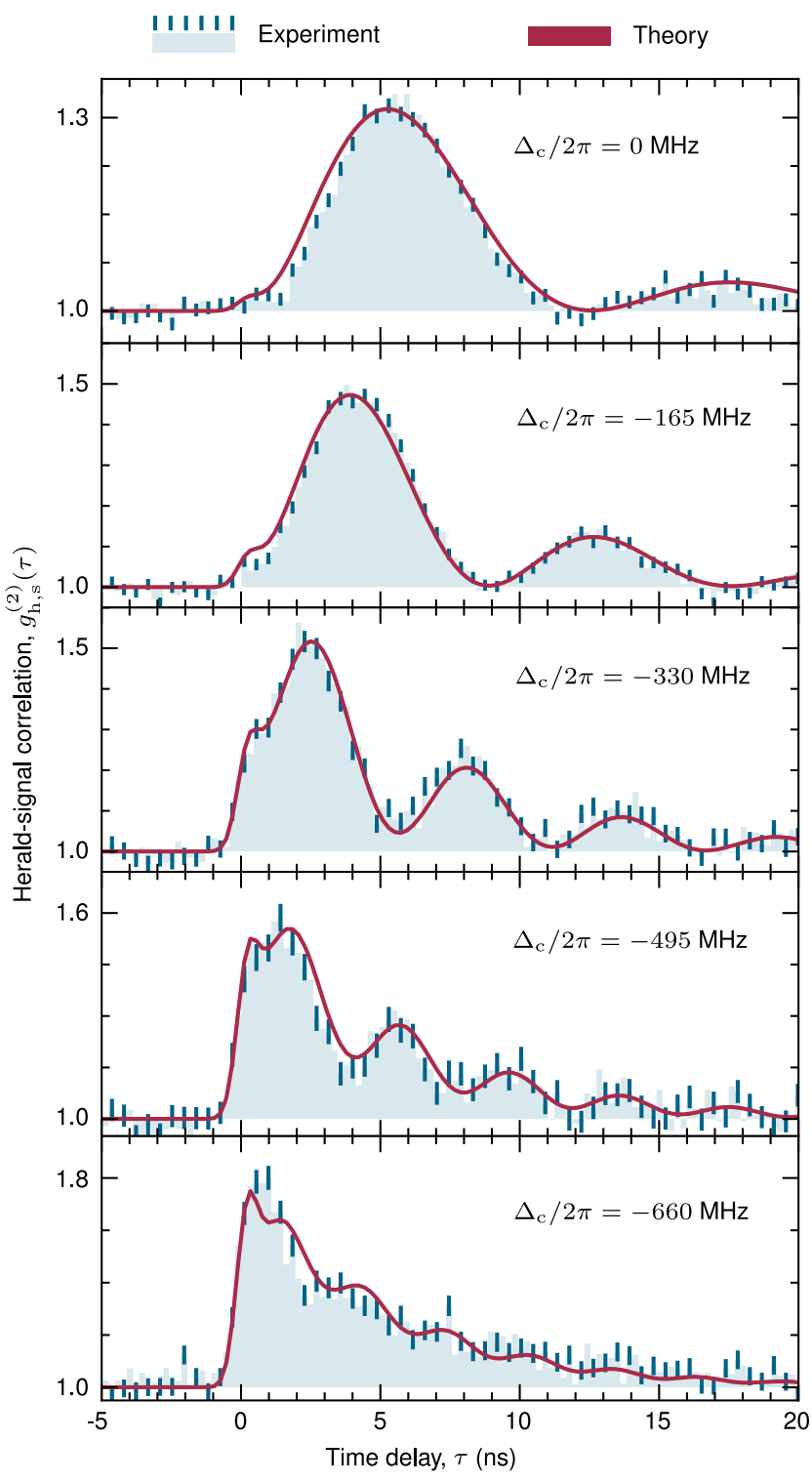

FIG. 3. The persistence of coherence between two collective excitation components. Experimental data (blue) showing interference resulting from the coherent splitting of a single excitation across two groups of atoms with relative motion. The Doppler shift leads to beats in the state readout with a frequency proportional to the relative velocity. The detuning of a strong dressing laser, $\Delta_{c}$, sets the velocities of the excited atoms and thereby determines the beat frequency. A theoretical model (red curves) finds excellent agreement with the data across the entire range of detunings studied. The error bars on the experimental data are calculated assuming Poissonian noise on the individual histogram bins [38].

field. Such a two-color photon may be a useful resource for entangling two spatially separated atomic quantum memories. In such a scheme, each memory would absorb one part of the two-color photon. A symmetric resource state can be prepared by resonant driving $\left(\Delta_{c}=0\right)$ that symmetrically excites two velocity classes, moving in opposite directions 
with velocities $\pm \Omega_{c} /(k \sqrt{8})$ set by the coupling laser power through $\Omega_{c}$. Similar diamond schemes in rubidium would allow for the generation of telecom-wavelength single photons [43].

In conclusion, excellent agreement between the theory and experiment demonstrates that atoms in strongly dressed thermal vapors [44] offer a reliable platform for quantumstate engineering. The addition of external magnetic fields allows for the selective excitation and observation of welldefined simple systems that can be completely and accurately modeled $[19,20]$. The collective excitation of two velocity groups is an example of an entangled state that is robust against single-atom loss and dephasing [45]. With the emission of two-color heralded single photons providing a direct relative phase measurement and tunability of the atomic response through adjustments to the dressing laser, these states can be further explored in protocols for the quantum-state control of atoms and light.

The data sets generated and/or analyzed during the current study are available in the Durham University Collections repository [46].

We thank K. J. Weatherill, E. Bimbard, R. S. Mathew, and $\mathrm{H}$. Busche for their helpful and informative comments. We acknowledge financial support from EPSRC (Grant No. EP/L023024/1) and Durham University, UK. C. S. A. is supported by the European Union (EU) Project No. H2020-FETPROACT-2014 184 Grant No. 640378 (Rydberg Quantum Simulators, RySQ).

*daniel.whiting@durham.ac.uk

[1] K. Hammerer, A. S. Sørensen, and E. S. Polzik, Rev. Mod. Phys. 82, 1041 (2010).

[2] A. MacRae, T. Brannan, R. Achal, and A. I. Lvovsky, Phys. Rev. Lett. 109, 033601 (2012).

[3] C. W. Chou, S. V. Polyakov, A. Kuzmich, and H. J. Kimble, Phys. Rev. Lett. 92, 213601 (2004).

[4] D. J. Saunders, J. H. D. Munns, T. F. M. Champion, C. Qiu, K. T. Kaczmarek, E. Poem, P. M. Ledingham, I. A. Walmsley, and J. Nunn, Phys. Rev. Lett. 116, 090501 (2016).

[5] M. Hosseini, G. Campbell, B. M. Sparkes, P. K. Lam, and B. C. Buchler, Nat. Phys. 7, 794 (2011).

[6] A. I. Lvovsky, B. C. Sanders, and W. Tittel, Nat. Photonics 3, 706 (2009).

[7] L.-M. Duan, M. D. Lukin, J. I. Cirac, and P. Zoller, Nature (London) 414, 413 (2001).

[8] M. Fleischhauer and M. D. Lukin, Phys. Rev. Lett. 84, 5094 (2000).

[9] J. D. Pritchard, K. J. Weatherill, and C. S. Adams, in Annual Review of Cold Atoms and Molecules, edited by K. W. Madison, Y. Wang, A. M. Rey, and K. Bongs (World Scientific, Singapore, 2013), pp. 301-350.

[10] T. D. Ladd, F. Jelezko, R. Laflamme, Y. Nakamura, C. Monroe, and J. L. O'Brien, Nature (London) 464, 45 (2010).
[11] J. Kitching, S. Knappe, and L. Hollberg, Appl. Phys. Lett. 81, 553 (2002).

[12] T. H. Sander, J. Preusser, R. Mhaskar, J. Kitching, L. Trahms, and S. Knappe, Biomed. Opt. Express 3, 981 (2012).

[13] J. A. Sedlacek, A. Schwettmann, H. Kübler, R. Löw, T. Pfau, and J. P. Shaffer, Nat. Phys. 8, 819 (2012).

[14] S. Brandt, A. Nagel, R. Wynands, and D. Meschede, Phys. Rev. A 56, R1063 (1997).

[15] D. Budker and M. Romalis, Nat. Phys. 3, 227 (2007).

[16] M. V. Balabas, T. Karaulanov, M. P. Ledbetter, and D. Budker, Phys. Rev. Lett. 105, 070801 (2010).

[17] A. Sargsyan, D. Sarkisyan, U. Krohn, J. Keaveney, and C. Adams, Phys. Rev. A 82, 045806 (2010).

[18] A. Sargsyan, Y. Pashayan-Leroy, C. Leroy, and D. Sarkisyan, J. Phys. B 49, 075001 (2016).

[19] D. J. Whiting, J. Keaveney, C. S. Adams, and I. G. Hughes, Phys. Rev. A 93, 043854 (2016).

[20] D. J. Whiting, E. Bimbard, J. Keaveney, M. A. Zentile, C. S. Adams, and I. G. Hughes, Opt. Lett. 40, 4289 (2015).

[21] O. Firstenberg, M. Shuker, A. Ron, and N. Davidson, Rev. Mod. Phys. 85, 941 (2013).

[22] S. Haroche, in High-Resolution Laser Spectroscopy, edited by K. Shimoda (Springer-Verlag, Berlin, 1976), pp. 253-313.

[23] S. Clemmen, A. Farsi, S. Ramelow, and A. L. Gaeta, Phys. Rev. Lett. 117, 223601 (2016).

[24] P. Treutlein, Physics 9, 135 (2016).

[25] L. Weller, K. S. Kleinbach, M. A. Zentile, S. Knappe, C. S. Adams, and I. G. Hughes, J. Phys. B 45, 215005 (2012).

[26] M. A. Zentile, R. Andrews, L. Weller, S. Knappe, C. S. Adams, and I. G. Hughes, J. Phys. B 47, 075005 (2014).

[27] R. T. Willis, F. E. Becerra, L. A. Orozco, and S. L. Rolston, Phys. Rev. A 82, 053842 (2010).

[28] B. Srivathsan, G. K. Gulati, B. Chng, G. Maslennikov, D. Matsukevich, and C. Kurtsiefer, Phys. Rev. Lett. 111, 123602 (2013).

[29] B. Zhao, Y.-A. Chen, X.-H. Bao, T. Strassel, C.-S. Chuu, X.-M. Jin, J. Schmiedmayer, Z.-S. Yuan, S. Chen, and J.-W. Pan, Nat. Phys. 5, 95 (2009).

[30] S. Haroche, J. A. Paisner, and A. L. Schawlow, Phys. Rev. Lett. 30, 948 (1973).

[31] A. Aspect, J. Dalibard, P. Grangier, and G. Roger, Opt. Commun. 49, 429 (1984).

[32] C. G. Wade, N. Šibalić, J. Keaveney, C. S. Adams, and K. J. Weatherill, Phys. Rev. A 90, 033424 (2014).

[33] M. Gross, J. M. Raimond, and S. Haroche, Phys. Rev. Lett. 40, 1711 (1978).

[34] S. Du, J. Wen, and M. H. Rubin, J. Opt. Soc. Am. B 25, C98 (2008)

[35] S.-J. Yang, X.-H. Bao, and J.-W. Pan, Phys. Rev. A 91, 053805 (2015).

[36] See Supplemental Material at http://link.aps.org/ supplemental/10.1103/PhysRevLett.118.253601 for a derivation of the theoretical model for $g_{h, s}^{(2)}$ and additional information regarding the energy level scheme and the filtering of the herald and signal photons. 
[37] R. J. Glauber, Phys. Rev. 130, 2529 (1963).

[38] I. G. Hughes and T. P. A. Hase, Measurements and Their Uncertainties: A Practical Guide to Modern Error Analysis (Oxford University, Oxford, 2010).

[39] B. Huber, A. Kölle, and T. Pfau, Phys. Rev. A 90, 053806 (2014).

[40] F. Ripka, Y.-H. Chen, R. Löw, and T. Pfau, Phys. Rev. A 93, 053429 (2016).

[41] J. Wen, S. Du, Y. Zhang, M. Xiao, and M. H. Rubin, Phys. Rev. A 77, 033816 (2008).
[42] Y. O. Dudin, L. Li, and A. Kuzmich, Phys. Rev. A 87, 031801 (2013).

[43] R. T. Willis, F. E. Becerra, L. A. Orozco, and S. L. Rolston, Opt. Express 19, 14632 (2011).

[44] N. Šibalić, J. M. Kondo, C. S. Adams, and K. J. Weatherill, Phys. Rev. A 94, 033840 (2016).

[45] W. Dür, G. Vidal, and J. I. Cirac, Phys. Rev. A 62, 062314 (2000).

[46] See http://dx.doi.org/10.15128/r19c67wm81t. 